\title{
Have You Met Your METs? - Enhancing Patient Motivation to Achieve Physical Activity Targets in Cardiac Tele-rehabilitation
}

\author{
Supraja Sankaran \\ Hasselt University-tUL-Expertise \\ Center for Digital Media \\ Diepenbeek, Belgium \\ supraja.sankaran@uhasselt.be
}

\author{
Kris Luyten \\ Hasselt University-tUL- Flanders Make \\ Expertise Center for Digital Media \\ Diepenbeek, Belgium \\ kris.luyten@uhasselt.be
}

\author{
Dominique Hansen \\ Hasselt University- REVAL-BIOMED \\ Heart Center, \\ Hasselt, Belgium \\ dominique.hansen@uhasselt.be
}

\author{
Paul Dendale \\ Hasselt University- Faculty of Medicine and Life Sciences; \\ Department of Cardiology, Jessa Hospital, \\ Hasselt, Belgium \\ paul.dendale@uhasselt.be
}

\author{
Karin Coninx \\ Hasselt University-tUL-Expertise Center for Digital Media \\ Diepenbeek, Belgium \\ karin.coninx@uhasselt.be
}

\begin{abstract}
Physical exercise is a primary component of cardiac rehabilitation. Interpreting exercise targets and being aware of the expected effort while rehabilitating independently at home is challenging for patients. Our work aims to assist cardiac patients in understanding their condition better and motivating them to achieve their exercise targets in a tele-rehabilitation setting. We introduce a patient-centric intelligible visualization approach to present prescribed rehabilitation targets to patients based on Metabolic Equivalent of Tasks (METs). We assessed efficacy of intelligible visualizations on patients' comprehension in a lab study. We evaluated the impact on patient motivation and health outcomes in field studies. Patients were able to manage their prescribed activities, monitor their progress, and gained understanding on how their physical activities contribute to their rehabilitation targets. Patients confirmed that the intelligible visualizations motivated them to achieve their targets better. We observed an improvement in overall physical activity levels and health outcomes of patients.
\end{abstract}

Patient-centred computing, Self-management, Patient Motivation, Secondary Prevention, Tele-rehabilitation, Intelligibility, Self-awareness

\section{INTRODUCTION}

Cardiac rehabilitation programs have proven to be effective to minimize recurrence and risk of disease, leading to reductions in risk of premature deaths (by about 40\%) and cost savings (Rauch et al., 2016). There are several studies that test the effectiveness and the applicability of home-based rehabilitation (tele-rehabilitation) approaches for various medical applications as summarized by Mampuya et al. (Mampuya, 2012). Physical activity is a core component of cardiac tele-rehabilitation. Conventional physical activity training programs in hospitals or specialized rehabilitation centres involve supervised exercise sessions that are guided and monitored by physiotherapists (Mampuya, 2012). Continuing with exercising independently at home is crucial in order to avoid relapses and is often difficult for patients. In telerehabilitation, remote monitoring of physical activity to evaluate progress towards rehabilitation targets is challenging (Frederix et al., 2013; Hansen et al., 2010) as it relies on the willingness and the motivation of patients to perform physical exercises. We target patients suffering from Coronary Artery Disease (CAD) who have to continue their rehabilitation in the absence of supervised exercise training. For these patients, the challenge starts with interpreting their overall rehabilitation targets, identifying the types of physical activities they should do, and tracking their progress over time. For rehabilitation exercises, targets are more complex to determine than for general fitness exercises: patients are expected to exercise at a specific level of intensity and at 
appropriate heart rate zones for a predetermined duration. Therefore, it is important to make physical exercise prescriptions simple and understandable for patients, and to ensure easy self-monitoring of progress. Besides, it is also important that the process is not cumbersome and helps in enhancing motivation in patients.

In our work, we applied the design principle of intelligibility to support patient self-awareness, comprehension and in enhancing motivation. An intelligible system allows users to understand and even learn about the inner working of the system. It has been shown that intelligible systems are easier to use and are more trusted by their users (Lim and Dey, 2013). In order to add credibility to systems that are designed to support rehabilitation, it becomes even more essential for patients to be able to trust the system. The prevalent method to communicate physical exercise tasks is the medical professional orally communicating it to the patient or writing these on a prescription paper during a consultation. Lack of a clear and standardized way to describe and structure this information makes it difficult for the patient to interpret the exercise targets correctly and hinders communication between the medical staff and the patient. Given the complexity of this domain and the importance of a correct approach for our target group, we involve different expertise domains (cardiologists, physiotherapists and computer scientists) to guide the research, design, and development work presented in this paper. Additionally, we draw upon other systems in literature that focus on comprehensive approaches to rehabilitation and integration of persuasive design principles (Sankaran et al., 2016; Tavella et al., 2015).

In this paper, we focus on optimizing the representation of physical activity for patients, as it is the core component in cardiac rehabilitation. We describe how we integrated aspects of intelligibility, alongside persuasive principles, to make patients more self-aware of their targets and progress. We present the insights gathered from three studies to validate the efficacy of these aspects. In an initial lab study, we evaluated how the intelligible visualizations supported patients in becoming more self-aware in comprehending their rehabilitation targets. We further assessed the impact on enhancing patients' motivation to achieve their goals in a 4-week field study using a prototype mobile application. Finally, we evaluated the impact of their self-awareness and motivation in increasing physical activity levels and health outcomes in an 8-week clinical study. Based on these outcomes, we can ascertain that using persuasive design and intelligible visualizations to make patients more self-aware can contribute in persuading them to achieve their rehabilitation targets. Additionally, we observe that the nudges triggered via the application increases overall physical activities performed by patients, thereby creating a positive health behaviour.

\section{MAKING EXERCISE PRESCRIPTIONS INTELLIGIBLE}

It is essential for patients to understand and grasp relevant information from the exercise prescription. We target cardiac patients, who are mostly 50 years or older and may have a fear of exercising (kinesiophobia). Moreover, most of them are unaccustomed to non-supervised exercise training due to prolonged sedentary lifestyle and lack of proper guidance after their hospital-based rehabilitation. Therefore, it is critical that their interpretation of the prescription and progress is precise. Supporting personalization of prescriptions and designing comprehensible information representation on a mobile device is essential for this target group of patients. O'Kane et al. (O'Kane and Mentis, 2012) emphasize on the important difference between information needs of clinicians and patients, where patients need an interpretation of what it means for their lives. The core design principle we use to accomplish this through our user interface is intelligibility (Bellotti and Edwards, 2001). Through appropriate usage of this design principle, we can make patients more self-aware of their condition and progress, which can in turn persuade them to achieve and even surpass their rehabilitation targets.

We contribute to increasing patients' selfawareness on their progress by focusing on the following aspects of intelligibility:

(i) Visualizations that support correct interpretation and understanding of how progress is tracked.

(ii) Feedback on and prediction of progress.

(iii) Tailored visualizations based on patient profile.

\subsection{Correct Interpretation and Understanding}

Visualizing all components of an exercise prescription in a simple non-overwhelming manner for patients is not trivial. The user interface should make patients aware of what activities contribute to their rehabilitation targets, and how much they contribute in different settings (e.g. longer exercise sessions or increasing the intensity or frequency of exercise training). This implies that the interface visualization needs to train the patient to make reasonable estimates of their efforts and progress. We worked together with patients, cardiologists, and physiotherapists to iteratively design and improve our visualizations. When dealing with visual representation for a target group consisting of patients, it is essential to focus on information 
visualization rather than scientific visualization such as complex graphs or medical charts (Faisal et al., 2012). For example, in this study, we use an animated character running towards the target $\left(z^{*}\right)$ to visualize the progress patients make as shown in Figure 1.a. This makes the idea behind the progress bar more perceptible than using just bar charts or graphs as is the case in most applications available for monitoring physical activity (Walters et al., 2010). To enable patients to make sense of personal health information, it is necessary to exploit the patient's perceptions to present targets and prescriptions (Lim et al., 2016).

Figure 1.a shows a representation of the prescription in the mobile application interface. An exercise prescription for rehabilitation describes the type, intensity, frequency, and duration of exercises. For example, the patient would be advised to indulge in moderately intense physical activities up to 5 days a week for 40 to 60 minutes per exercise session. To simplify it for patients, we combined these parameters into a unified metric, the Metabolic Equivalent of Tasks (METs represented as kilocalories) using guidelines of ACSM (American College of Sports Medicine) for exercise prescription and testing (Swain et al., 2014) as detailed in subsequent sections. This metric is proven to be precise and suited for measuring physical exercise volume (Jetté et al., 1990). In our work, we express this metric as a target score for patients. Given that intensity, frequency and duration are represented as range (e.g. moderate- high intensity for 40-60 minutes per session); the final targets are also presented to patients as a range (minimal and maximal targets). Patients are free to choose the type of physical activity they wish to do from an extensive list of activities. This list is curated by medical experts, and contains activities that contribute to achieving their rehabilitation targets. Two flags are used to visualize the minimal $(\curvearrowright)$ and maximal $(\bowtie)$ target values.

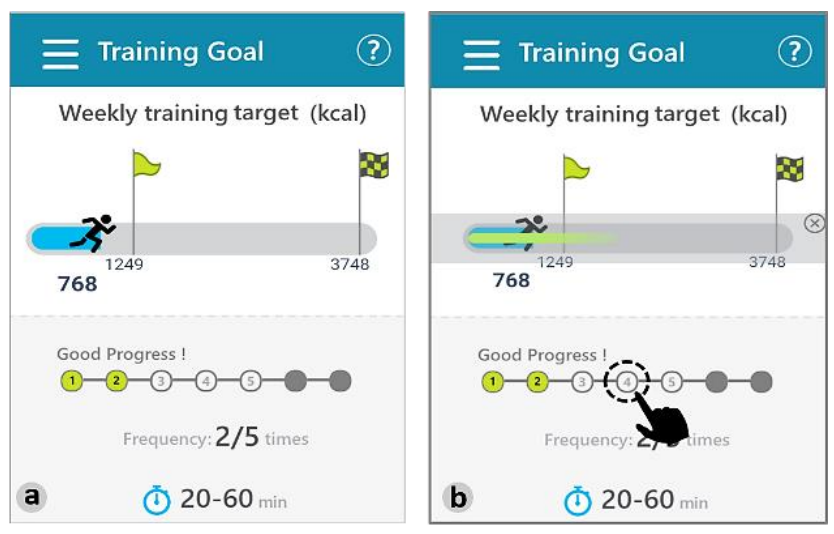

Figure 1. (a) Screenshot from the application that shows prescription parameters, targets and progress. (b) Representation of progress prediction upon selecting a future session.
The finish flag ( $\otimes$ ) subtly nudges the patient to aim for the maximal achievement and not stop exercising after reaching the minimal target $(\Vdash)$. This is validated by field tests presented in later sections. Frequency and duration (॰) of physical activities are visualized numerically. Apart from these elements, the number of exercise sessions completed by the patients and the ones yet to be done can also be easily consulted in the visualization of session progress ((2-3-O) and the completed frequency. Patients also get motivating textual feedback above the session progress visualization such as- 'keep going' or 'good progress'. Despite the apparent simplicity of the prescription display, the two flags provide a better insight in the rehabilitation progress. Our representation is different from the goal setting and visualization in most fitness and physical activity applications (Stawarz et al., 2015): using a target range instead of a singular goal allows patients to self-manage their target achievement, instead of feeling pressurized to achieve one single goal. The results of our field studies confirm that some patients strived to reach the maximal target or go beyond it, while other patients stopped after reaching their minimal target.

\subsection{Progress Feedback and Prediction}

Quantifying exercise effort/capacity based on activities performed is challenging as it can largely vary between individuals. Methods used to collect this data can either be objective measurements obtained from monitoring devices (such as pedometers, accelerometers, metabolic carts etc.) or subjective values that are self-reported by the user (such as diaries, questionnaires, surveys etc.). We collected self-reported activity logs on the app and data using IPAQ (International Physical Activity Questionnaire) (Kurtze et al., 2008). We obtained objective measurements on exercise capacity using ergo spirometry.

A vast literature survey of 293 articles on selfreported against measured values (Prince et al., 2008) revealed the need for valid, accurate, and reliable measures of physical activity. A known major shortcoming of subjective approaches is their heavy reliance on an individual's recollection of events and knowledge of what is the definition of exercise training/physical activity. Therefore, depending on the context, such approaches may be prone to under- or over-reporting of physical activity volume/intensity (Celis-Morales et al., 2012). On the other hand, there is also evidence suggesting barriers of engaging with objective measurements such as activity monitors (e.g., discomfort, hardware issues, or drop in usage over time) (Harrison et al., 2015). Therefore, it is essential to find a balance between both means of measurements and identify an approach that can work in both contexts. 
We use a dynamic mapping that, based on the patient profile, calculates personalized METs scores achieved per exercise. The METs target evolves when the patient's exercise capacity improves. Studies suggest that no single technique can be used to gather exercise capacity and energy expenditure information and it is recommended to use multiple complementary methods (Altini et al., 2013; Schutz et al., 2001). This is critical in evaluating current and changing physical activity levels, physical activity interventions, and the relationships between physical activity and rehabilitation outcomes.

Feedback on progress gently nudges them to increase their efforts when appropriate. An important feature here is that patients can consult their future training efforts in advance: their predicted progress is shown when tapping on a 'yet-to-be-completed' exercise session (Figure 1.b). When users tap on a future session number, the predicted progress is visualized as an overlay on top of the existing progress. The cumulative effort of the completed exercise sessions is used to compute the predicted progress, as we currently do not ask patients to plan a specific exercise type. Since the prediction is essentially an estimate based on the previous effort and not a precise value, the prediction visualization is shown as a bar that fades out towards the end.

The extent of fade-out gradient is based on the number of exercise sessions completed. For example, if the patients have done only one session out of 5 , the extent of fade out is greater since the subsequent activities they will be performing can have a large variation in energy expenditure. Our system uses the completed exercise sessions to calculate the current exercise capacity of the patient.

\subsection{Dynamic Personalized Visualizations}

The visualizations are generated dynamically based on the personalized targets computed for every individual patient. The targets and parameters are configurable by physiotherapists through a remote dashboard application that integrates the validated EXPERT tool (Hansen et al., 2017). Patients are informed about the involvement of physiotherapists for their goal setting and with respect to progress monitoring. The position of the flags, the values, and the session progress visualizations are adapted accordingly. In addition, if patients' targets are modified during the course of the rehabilitation based on their progress, the visualizations are updated accordingly. This ensures that the visualisation presents the most recent state of the rehabilitation process, based on the physiotherapists' input as well as the progress they make during the rehabilitation program. Unlike fixed goals or user-defined goals in other fitness and behavior change apps (Stawarz et al., 2015), this method of gradually increasing targets based on the evolution of patients' condition over time helps in sustaining motivation and gradually nudging them to achieve their targets. This approach motivates patients that are unwilling to make a greater behaviour change by giving them achievable minimal targets to trigger them to be more physically active and sustain their motivation. Patients being aware of the involvement of physiotherapists in the process of tailoring targets further adds to the trust and credibility of the approach, thereby acertaining another aspect of intelligibility.

\section{TAILORING EXERCISE TARGETS AND MONITORING PROGRESS}

For the overall rehabilitation program to be successful, it is essential that patients have the ability and willingness to accommodate these prescribed exercise regimes in their everyday lives (Gjoreski et al., 2013). This implies that they need to feel motivated, in control and are accorded a sufficient degree of freedom in choosing what physical activities to perform, when and at what intensitiy. Campbell et al. (Campbell et al., 2001) reported that such exercise regimes can appear counter-productive to patients because of perceived ineffectiveness or discomfort they may experience. Patients often struggle to understand the progress they make through their rehabilitation program, because of a lack of feedback on their progress. A rehabilitation program, if done right and in absence of unexpected health incidents, gradually improves a patients' exercise capacity and overall health condition. Yet, observing improvements in their condition over time is difficult for patients (Harrison et al., 2015) - especially when the goal setting is not adapted according to these improvements. We break down the overall goal into weekly targets (micro-goals), as this is a custom practice by physiotherapists in conventional hospital-based rehabilitation. These weekly targets are gradually adapted based on patients' progress over time and allow patients to see their overall progress. In this section, we describe in detail the process adapted by physiotherapists to tailor exercise prescriptions and monitor progress of the patients. The physiotherapists do this using a remote caregiver dashboard interface that dynamically communicates with the patients' mobile application.

\subsection{Tailoring Exercise Targets}

The key underlying principle behind calculating the tailored micro-goals for patients is the "Frequency, Intensity, Time, and Type" (FITT) principle (Clinical Exercise Physiology, 2013). In our approach, the process of prescribing exercise training is divided into three steps: 
Step 1: Assess the current health and fitness condition of patients. To get this information, an ergo-spirometry test (a monitored biking effort in a lab setting) is done at the hospital. This non-invasive and objective method can generate an accurate assessment of cardiopulmonary functions and metabolism of an individual. The result of this test helps us establish preliminary estimates of maximal thresholds of exercise capacity for every patient.

Step 2: Determine individual risk factors and exercise capacity. Based on the threshold values obtained in the previous step, a physiotherapist determines the individual risk factors and the exercise capacity of every patient.

Step 3: Formulate the exercise training prescription. Based on the determined exercise capacity, the physiotherapist establishes the frequency, intensity, and time of training using the EXPERT tool (Hansen et al., 2017). This tool enables the physiotherapists to further tailor the FITT-based prescription as per individual patient pathologies and risk factors. Using the mobile application, patients are free to choose the type of activity they wish to perform and that fit the prescribed intensity levels. An activity-specific MET value that corresponds to the prescribed exercise intensity (Vanhees et al., 2012) is used together with activity duration and patient parameters to compute the targets represented by the flags. These computed targets are configured individually for every patient in the application.

\subsection{Monitoring Progress}

The individually prescribed targets, presented as weekly training scores, essentially refer to workloads determined by the amount of energy expenditure that needs to be achieved in a week. Our approach to determine the progress is divided into four key steps:

Step 1: Identify METs based on activity performed. We do this using the Compendium of Physical Activities (Ainsworth et al., 2000).

Step 2: Factor for an individual's body mass and resting metabolism (also known as "idle burn", the amount of energy expended when a person is sedentary or at rest). We use the following formula to calculate energy expenditure (in kcal per minute) derived from the widely used ACSM's guidelines for exercise testing and prescription as explained below (Equation 1).

\section{$($ METs $\times 3.5 \times$ body mass in $\mathrm{kg}) / 200$ = Energy Expenditure in kcal - $\min ^{-1}$}

Where, $3.5 \mathrm{~mL} \cdot \mathrm{kg}^{-1} \cdot \mathrm{min}^{-1}$ is the standard factor for a resting energy expenditure.

Equation 1. Formula for calculating personalized energy expenditure including idle burn.
Step 3: The energy expenditure is converted into gross and net expenditure by multiplying the duration and frequency of selected activities (Equation 2).

Step 4: As a final step, the net energy expenditure that is acquired from different activities is cumulated to obtain the total expenditure (Equation 2). This total energy expenditure is expressed as a single score to quantify the progress made by the patient.

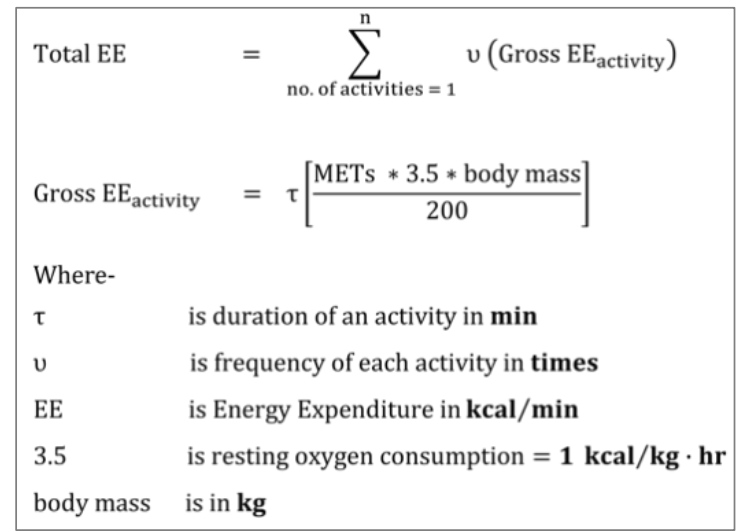

Equation 2. Formula for computing personalized energy expenditure during different activities.

The total energy expenditure score that results from Step 4 is used in the progress visualization in both the caregivers' dashboard interface and the patients' application interface. Presenting the score as a number (in kcals) in the user interface ensures that users also get to see fine-grained feedback on their progress. A caloric representation enables patients to not just relate their goals to physical activity but also to diet instructions they receive during rehabilitation. It also helps them to gain insight on the contribution of specific activities (including their intensity and duration) towards their goals. Furthermore, since the target scores shown underneath the two flags can also change over time, users are also informed about the evolution of their exercise capacity, i.e. a better exercise capacity implies a higher maximal target.

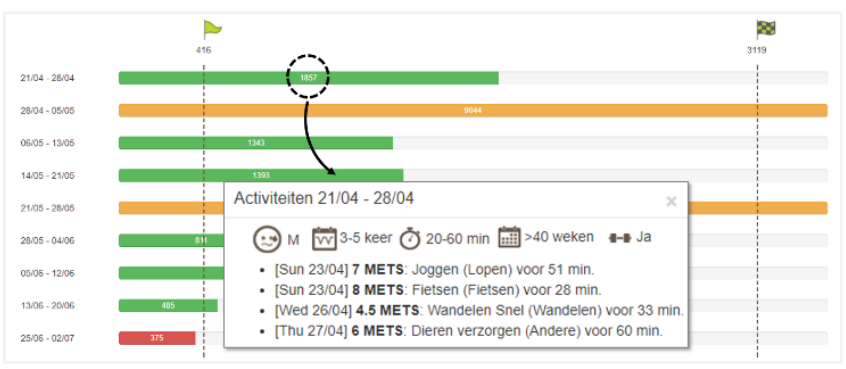

Figure 2. Screenshot of the caregiver's dashboard interface showing the weekly progress of a patient with respect to prescribed targets.

The caregivers or therapists can follow-up the progress of a patient remotely using the remote caregiver dashboard interface, shown in Figure 2. 
They can further check individual activities performed by the patient by clicking on each week's progress in comparison to what was recommended. All these elements facilitate physiotherapists to more precisely tailor targets and adapt them better based on individual patients' exercise patterns.

\section{EVALUATIONS}

To gain insights on patients' understanding of rehabilitation progress, the impact of their understanding and self-awareness in enhancing motivation to achieve targets, and its influence on their activity levels, we conducted three studies (presented in Error! Reference source not found.). The ethical committees of Jessa Hospital and Hasselt University, Belgium, approved these studies. Patients signed an informed consent during start-up and could quit the study at any stage. None of the patients from an earlier study was included in the subsequent studies. All patient data procured, used and analysed during the various studies were handled anonymously and confidentially. All data was stored at a dedicated encrypted server at our research department and only the primary investigators had access to this data.

\subsection{Evaluating intelligibility}

\subsubsection{Process}

This study was conducted in a lab setting. The study was scenario-driven with an aim to (a) gather immediate insights regarding patients' understanding on how they perceive this approach in different task scenarios, and (b) observe their subsequent interpretations and understanding as the scenarios evolved. Gaining detailed knowledge on their understanding of different scenarios would have been difficult in the field. Participants were presented with five realistic scenarios based on situations they may encounter during their rehabilitation process. The scenarios included tasks on interpreting prescription parameters, logging activities on the app with different frequencies, duration and intensity levels, and investigating a future situation using progress prediction based on performed activities. Participants were encouraged to think aloud while performing different tasks. A camera was positioned to record their interaction with the application while recording the audio and video. As the participant went through the tasks, the observer took notes. Each test session was concluded by a semi-structured interview to gain insights into their overall understanding, usefulness and perception of the intelligible visualization.

\subsubsection{Findings}

For each of these aspects (their interpretation of the represented parameters, perceived usefulness and the presentation of the prescription), they were asked to give a score on 10. Presentation of parameters and usefulness of these visualizations received an average score of 7 and the interpretation of prescription got an average score of 8 (Figure 3).

Table 1. Overview of setup, evaluations and data collected during the studies

\begin{tabular}{|c|c|c|}
\hline $\begin{array}{l}\text { Evaluating intelligibility } \\
\text { (In-situ study) }\end{array}$ & $\begin{array}{l}\text { Evaluating impact on motivation } \\
\text { (Field study) }\end{array}$ & $\begin{array}{c}\text { Evaluating physical activity levels } \\
\text { (Field study) }\end{array}$ \\
\hline 8 CAD patients (PL1-PL8) & 5 CAD patients (PF1- PF5) & 32 CAD patients (PC1-PC32) \\
\hline $\begin{array}{l}\text { Undergoing rehabilitation at the } \\
\text { hospital + independently rehabilitating } \\
\text { at home }\end{array}$ & $\begin{array}{l}\text { Undergoing rehabilitation at the } \\
\text { hospital + independently rehabilitating } \\
\text { at home }\end{array}$ & $\begin{array}{l}\text { Finished rehabilitation at the hospital + } \\
\text { independently rehabilitating at home }\end{array}$ \\
\hline Study Duration: 1 hour & Study duration: 4 weeks & Study duration: 8-10 weeks \\
\hline $\begin{array}{l}\text { Evaluated aspects: } \\
\text { - Understandability of visualizations } \\
\text { - Usefulness in telerehabilitation } \\
\text { - Presentation of targets }\end{array}$ & $\begin{array}{l}\text { Evaluated aspects: } \\
\text { - Understandability of visualizations } \\
\text { - Usefulness in telerehabilitation } \\
\text { - Presentation of targets } \\
\text { - Impact on motivation }\end{array}$ & $\begin{array}{l}\text { Evaluated aspects: } \\
\text { - Impact of various elements on } \\
\text { motivation } \\
\text { - Change in physical activity levels } \\
\text { - Other health outcomes }\end{array}$ \\
\hline Data Collection: & Data Collection: & Data Collection: \\
\hline $\begin{array}{l}\text { Start-up: Elicitation questionnaire } \\
\text { During study: task scenarios } \\
\underline{\text { End: semi-structured interview }}\end{array}$ & $\begin{array}{l}\text { Start-up: Elicitation questionnaire } \\
\text { During study: usage and activity logs, } \\
\text { diary } \\
\underline{\text { End: semi-structured interview }}\end{array}$ & $\begin{array}{l}\text { Start-up: Elicitation questionnaire, } \\
\text { IPAQ, physiological parameters } \\
\text { During study: usage and activity logs; } \\
\text { followed up via caregiver dashboard } \\
\text { application } \\
\text { End: semi-structured interview, IPAQ, } \\
\text { physiological measurements }\end{array}$ \\
\hline
\end{tabular}


While for some participants everything was clear right away, others acknowledged that they struggled to interpret the difference between two flags (minimal and maximal target) in the beginning but it got clearer as they progressed through different scenarios. Patients were able to interpret the impact on progress of variations in activities, duration and intensities. This finding implies that our intelligible visualization can facilitate in increasing selfawareness of patients. Most participants also found it useful to track their progress in this manner.

\begin{abstract}
"Yes, this way you have an overview. This way you see that you have to be active. You see that you need to have a good workout. Otherwise you would always write it down (referring to different prescription elements) which will give you a lesser overview with respect to your goals". [PL2]
\end{abstract}

\subsection{Evaluating Impact on Motivation}

After we iterated on the feedback collected from the lab test, we conducted a field test to assess how patients' perception of their condition evolved, and to evaluate the efficacy of the intelligible prescription visualization on increasing selfawareness and sustaining motivation over a longer period. In the field test, alongside the physical activity and training goal modules, patients used different modules of the mobile application corresponding to other rehabilitation components. In addition to evaluating patients' motivation over the span of 4 weeks when interacting with these visualizations, this allowed us to evaluate whether our intelligible visualization also functions in a broader self-management application context with a more holistic rehabilitation approach.

\subsubsection{Process}

Participants used the mobile application to record activities performed at the rehabilitation centre, activities performed independently at home, their medication intake, and other physiological parameters such as weight and blood pressure (if they had the appropriate measuring devices). Participants also received a paper-based diary to record their feedback each week on two criteria using a 5-point Likert scale (from strongly disagree to strongly agree). The two criteria were (1) to verify how viewing progress with respect to the goal motivated them to exercise more and (2) checking if the estimated prediction was helpful to assess whether their efforts were sufficient to reach their goals. At the end of the study duration, they were invited for a semi-structured interview to gather insights on the influence of using this application on motivation, their overall understanding of their targets and progress, the usefulness of using such an application, and other general perceptions about the application.

\subsubsection{Findings}

In terms of understandability, usefulness and overall perception, the results were comparable to the earlier lab study on assessing intelligibility. The average score for usefulness and presentation of prescription parameters increased from 7 to 8 , suggesting that patients find it useful after having used it in a real-life context (Figure 3). In terms of motivation, all participants perceived this approach of viewing their weekly targets, and the progress they make against it, as motivating. All patients found the visualization of a man running towards the goal ( $\left.3^{*}\right)$ motivating and felt the 'nudge' to keep progressing towards the flags (target). Some patients also said that the session progress visualization (2-2-0) and the textual feedback additionally contributed to their motivation as they felt a sense of achievement each time they completed a session. We also probed patients if the application triggered them to do more activities than their usual routine, to gain an initial understanding on how it might facilitate habit formation or long-term behaviour change. Two patients increased their activities to try to achieve their goal.

\section{"I saw that I did not make much progress even after completing all my 5 sessions (his target frequency). So I tried to do more to get as close to the flag as possible". [PF3]}

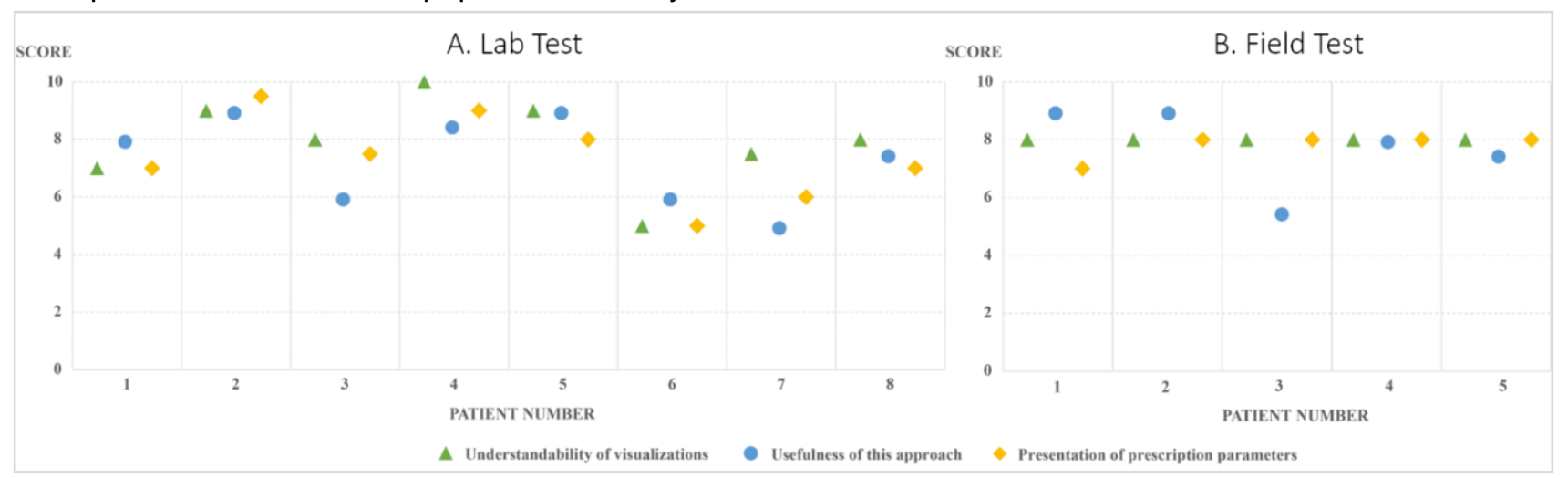

Figure 3. Results from initial lab and field studies on understandability, perceived usefulness and presentation of exercise prescription. 
One participant forgot to record his feedback using the paper-based diary. All other participants either 'agreed' or 'strongly agreed' (across all 4 weeks of the study) that viewing their progress motivated them to exercise more and the prediction was helpful to estimate if their efforts were sufficient. These findings reaffirm the efficacy of our intelligible visualization on promoting selfawareness in patients, thereby, persuading them in achieving their rehabilitation targets.

\subsection{Clinical Evaluation}

Integrated in an overall clinical evaluation of the mobile application, we assessed patients' physical activity levels along with overall medical improvements. In this paper, we focus on reporting on aspects related to our physical activity representation, such as physical activity levels and patients' motivation to achieve activity targets.

In this way, we further ascertain the positive outcomes of the lab study on intelligibility and the field study on the impact on motivation in the clinical evaluation. Of the 32 patients that were recruited, 4 patients had to quit the study after developing another health problem, 3 others did not use the app and one patient did not use the 'physical activity' module of the app. Therefore, the results presented are of the remaining 24 patients who used the app including its exercise target module and completed the study.

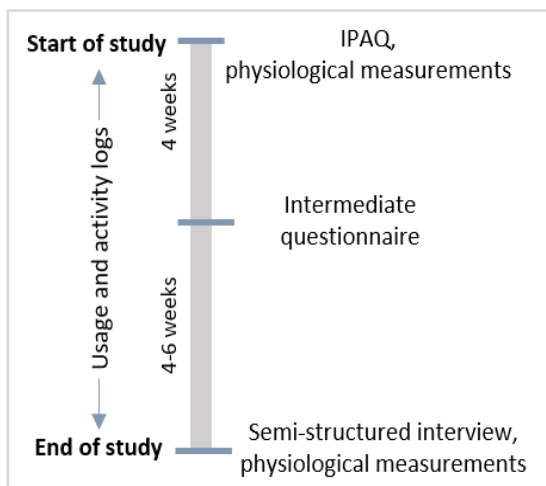

Figure 4. Timeline of the study (during the app usage phase) to evaluate the impact on physical activity levels and health outcomes.

\subsubsection{Process}

This clinical study was conducted as a cross-over randomized control trial where all participants were randomized into two groups on a 1:1 ratio. The total study was for a period of 4 months with a crossover point at 2 months (+/- 2 weeks). Patients randomized into group 1 used the app for the first two months and then received usual care for the subsequent two months. Patients in group 2 received usual care first and used the app in the second phase. In this manner, each patient used the app for a period of 8-10 weeks (Figure 4) and we were able to compare the progress and experience of all patients when using the app or in its absence. For the patients in group 1, we also gathered insights if the behaviour fostered by the app in the first phase was carried over to the second phase even in the absence of an active intervention using the app.

We measured patients' physiological measurements such as weight and blood pressure at start-up. Their exercise capacity (VO2max) was measured using a standardized ergo spirometry test. We also collected information on their physical activity behaviour over the week before start-up using the International Physical Activity Questionnaire (IPAQ). We collected usage and activity logs via the app. At the end of the first 4 weeks, we sent them an online questionnaire to assess the influence of specific features (visualization of targets, progress and prediction) on their motivation to achieve their goals. We followed up on these questions at the end of the study in individual semi-structured interviews with each patient.

\subsubsection{Findings}

In terms of understandability and the influence of progress and prediction in motivating patients to achieve their goals, the results were similar to the results of the field study (Figure 5). Most patients were positive about the influence of various app elements (visualization of goals, their progress and prediction) on their motivation to achieve prescribed goals (Figure 6).

"I really liked the fact that I could explore the effect of different activities on the goals! So I chose my activity sometimes based on the effect it would have on my progress towards the goals". [PC10]

$$
\begin{aligned}
& \text { Viewing prediction is very } \\
& \text { useful to know if my efforts } \\
& \text { are sufficient to achieve my } \\
& \text { goals. } \\
& \text { - Strongly disagree } \square \text { Disagree Neutral } \\
& \text { Agree } \quad \text { Strongly agree }
\end{aligned}
$$

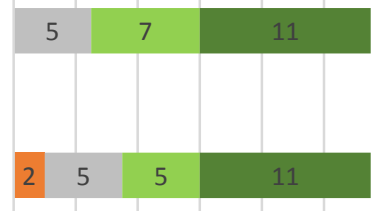

$\begin{array}{lllllll}0 & 4 & 8 & 12 & 16 & 20 & 24\end{array}$

Figure 5. Outcomes from the intermediate questionnaire at the end of 4 weeks of usage of the application (one patient forgot to fill the questionnaire; therefore, the data is missing).

The patients who reported that there was no extra motivation through using the app, attributed this to the fact that they already led an active lifestyle and 
only used the app to merely log their daily activities and not strive to increase their effort. These patients acknowledged that it was re-assuring to see their goals and progress and they feel more confident in logging it in the application since they know their therapists are following them up.

\footnotetext{
"..liked the motivational messages the app would give after entering a new activity. 'keep going', 'doing well'. I was already active, so did not do more. But was nice to see progress". [PC12]
}

We translated the data collected during start-up from the IPAQ questionnaire into METs scores based on IPAQ's scoring protocol (Uses and Instruments, 2005). We compared these to the mean METs score achieved by patients during the study. For $52 \%$ of patients there was an increase in mean METs as compared to baseline values. For the remaining patients, the baseline could not be considered owing to over-reporting or incomplete data. With respect to achieving the targets visualized in the app, $44 \%$ of the patients were mostly within the target range (between the minimal and maximal prescribed targets) and 52\% of patients mostly exceeded the maximal target. Only $4 \%$ of patients on average fell short of achieving the minimal target.

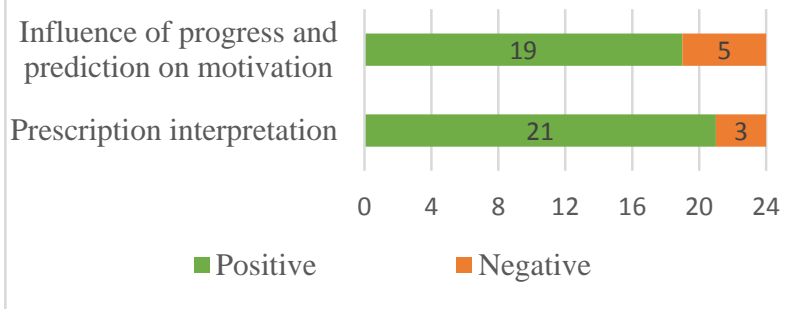

Figure 6. Patients' feedback on their interpretation of prescription parameters and the influence of the intelligible visualization on their motivation during the clinical evaluation.

In terms of health outcomes, we observed a minor increase in average VO2max (1.4\%) indicating increase in average exercise capacity of patients. We also observed an average reduction in weight $(0.6 \%)$ and blood pressure $(0.2 \%$ in systolic and $2.3 \%$ in diastolic blood pressure) indicating the positive influence of increased physical activity levels on other physiological parameters. Overall, we could see positive trends in target achievement rates and impact on other health outcomes.

\section{REFLECTIONS AND INSIGHTS}

In both the field and clinical tests, patients who were already leading an active lifestyle and met their physical activity targets mentioned that they did not really change their habits and continued to exercise as they do in their daily lives. The app or the intelligible visualization did not persuade them to increase their physical activity levels further. However, even these patients found that being able to see their activities contribute towards prescribed rehabilitation goals in the manner presented was re-assuring and gave them the confidence to sustain their current behaviour. This is also essential from a telerehabilitation perspective since studies report a decrease in progress of patients during tele-rehabilitation owing to lack of interest in the system and gradual decline in motivation over time (Patrick D. Savage, et al., 2012).

As with most persuasion approaches, there is a great dependence on an individual's willingness and attitude to change behaviour. We could also observe that in our studies where some patients were not willing to change behaviour even though they were fully aware of their health consequences. In that perspective, being remotely monitored by therapists 'compelled' a few patients to perform some activities just to be able to log it in the app.

"I did not pay much attention to it (referring to the part of being monitored), but I knew it was checked. So I took some effort to follow (referring to prescriptions) more strictly". [PC13]

We gathered that the majority of the patients either achieved their weekly targets or exceeded the maximal target. This certainly suggests a positive influence of the visualization and app on their overall rehabilitation process.

We also observed that some patients were not interested in active sports but instead chose to perform other activities such as dancing to achieve their goals. Therefore, giving users the option to choose various activities (as long as they met the intensity and duration constraints of the prescription) that can contribute to their targets also proved to facilitate in motivating them to reach their goals. For example, PC10 remarked-
"I don't like to sport and the app does not motivate me to do more sports. But I do move more in general because I want fill it in, and I am more consciously reflecting that $\mathrm{I}$ am moving. For instance, I would walk to the supermarket or ride my bicycle (instead of the car). Watching the man run to the flag (referring to the progress visualization) is super fun!"

Two patients were not comfortable with using technology in general and had no experience in using a smartphone. They remarked that while it was interesting to see their progress and log their activities, they would not continue using it over a long term since they find it quite challenging to cope and work with modern technology, which is often a common issue in our target population of patients. However, we can overcome this problem by automating most tasks, requiring minimal user input from patients. 
In terms of health outcomes, there was a promising indication that it may be possible to achieve a gradual improvement in overall health. However, a longer-term study with a larger group of participants is required to ascertain that claim which is beyond the scope of our study.

These insights establish that applying intelligibility alongside persuasive design can help in increasing patient self-awareness, enhancing and sustaining motivation and achieving rehabilitation targets; thereby, reducing dropout rates in tele-rehabilitation programs.

\section{CONCLUSION}

We presented a METs-based intelligible approach to visualize physical activity targets and progress in remote cardiac rehabilitation training. Intelligibility was brought in the design of the application to ensure that users understand and trust the user interface, and become more aware of their own performance. We focused on three core aspects of intelligibility to increase self-awareness of patients: (1) providing correct interpretation and understanding, (2) progress feedback and prediction, and (3) dynamic patient-centric visualizations. The intelligible visualizations support patients to have a correct perception and understanding of their physical exercise prescriptions as assessed in our lab study. It also enables patients to learn to make good estimates of their rehabilitation progress in different scenarios. This approach also enables caregivers and physiotherapists to precisely tailor targets, remotely monitor progress and accurately adapt prescriptions based on patients' progress and exercise behaviour. Being more self-aware of their progress and condition facilitates in keeping patients motivated while persuading them to achieve their physical activity rehabilitation targets as evaluated in our initial field study. The final clinical evaluation confirms that persuading patients to achieve their rehabilitation targets by increasing self-awareness using intelligible visualizations can help in increasing overall physical activity levels of patients.

\section{REFERENCES}

AINSWORTH, B., HASKELL, W., WHITT, M., IRWIN, M., 2000. Compendium of Physical Activities: an update of activity codes and MET intensities.

Altini, M., Penders, J., Vullers, R., Amft, O., 2013. Combining wearable accelerometer and physiological data for activity and energy expenditure estimation, in: Proceedings of the 4th Conference on Wireless Health - WH '13. ACM Press, New York, New York, USA, pp. 1-8.

https://doi.org/10.1145/2534088.2534106
Bellotti, V., Edwards, K., 2001. Intelligibility and Accountability: Human Considerations in Context-Aware Systems. Human-Computer Interact. 16, 193-212. https://doi.org/10.1207/S15327051HCl16234_ 05

Campbell, R., Evans, M., Tucker, M., Quilty, B., Dieppe, P., Donovan, J.L., 2001. Why Don't Patients Do Their Exercises? Understanding Non-Compliance with Physiotherapy in Patients with Osteoarthritis of the Knee. J. Epidemiol. Community Heal. 55, 132-138.

Celis-Morales, C.A., Perez-Bravo, F., Ibañez, L., Salas, C., Bailey, M.E.S., Gill, J.M.R., 2012. Objective vs. Self-Reported Physical Activity and Sedentary Time: Effects of Measurement Method on Relationships with Risk Biomarkers. PLoS One 7, e36345.

Clinical Exercise Physiology, 2013.

Faisal, S., Blandford, A., Potts, H.W.W., 2012. Making sense of personal health information: Challenges for information visualization. https://doi.org/10.1177/1460458212465213

Frederix, I., Driessche, N. Van, Hansen, D., Berger, J., Bonne, K., Alders, T., Dendale, P., 2013. Increasing the medium-term clinical benefits of hospital-based cardiac rehabilitation by physical activity telemonitoring in coronary artery disease patients. Eur. J. Prev. Cardiol. https://doi.org/10.1177/2047487313514018

Gjoreski, H., Kaluža, B., Gams, M., Milić, R., Luštrek, M., 2013. Ensembles of multiple sensors for human energy expenditure estimation, in: Proceedings of the 2013 ACM International Joint Conference on Pervasive and Ubiquitous Computing - UbiComp '13. ACM Press, New York, New York, USA, $p$. 359.

https://doi.org/10.1145/2493432.2493517

Hansen, D., Dendale, P., Coninx, K., Vanhees, L., Piepoli, M.F., Niebauer, J., Cornelissen, V., Pedretti, R., Geurts, E., Ruiz, G.R., Corrà, U., Schmid, J.-P., Vö Ller 35, H., Doherty, P., 2017. The European Association of Preventive Cardiology Exercise Prescription in Everyday Practice and Rehabilitative Training (EXPERT) tool: A digital training and decision support system for optimized exercise prescription in cardiovascular disease. Concept, . Eur. J. Prev. Cardiol. 24, 1017-1031. https://doi.org/10.1177/2047487317702042

Hansen, D., Dendale, P., Raskin, A., Schoonis, A., Berger, J., Vlassak, I., Meeusen, R., 2010. Long-term effect of rehabilitation in coronary artery disease patients: randomized clinical trial of the impact of exercise volume. Clin. 
Rehabil. 24, 319-327.

https://doi.org/10.1177/0269215509353262

Harrison, D., Marshall, P., Bianchi-Berthouze, N., Bird, J., 2015. Activity tracking, in:

Proceedings of the 2015 ACM International Joint Conference on Pervasive and Ubiquitous Computing - UbiComp '15. ACM Press, New York, New York, USA, pp. 617621.

https://doi.org/10.1145/2750858.2805832

Jetté, M., Sidney, K., Blümchen, G., 1990. Metabolic equivalents (METS) in exercise testing, exercise prescription, and evaluation of functional capacity. Clin. Cardiol. 13, 555565. https://doi.org/10.1002/clc.4960130809

Kurtze, N., Rangul, V., Hustvedt, B., 2008. Reliability and validity of the international physical activity questionnaire in the NordTrøndelag health study ( HUNT ) population of men 9, 1-9. https://doi.org/10.1186/14712288-8-63

Lim, B.Y., Dey, A.K., 2013. Evaluating Intelligibility Usage and Usefulness in a Context-Aware Application. Springer Berlin Heidelberg, pp. 92-101. https://doi.org/10.1007/978-3-64239342-6_11

Lim, C., Berry, A.B.L., Hirsch, T., Hartzler, A.L., Wagner, E.H., Ludman, E., Ralston, J.D., 2016. \&quot; It just seems outside my health\&quot; ; in: Proceedings of the 2016 ACM Conference on Designing Interactive Systems - DIS '16. ACM Press, New York, New York, USA, pp. 1172-1184. https://doi.org/10.1145/2901790.2901866

Mampuya, W.M., 2012. Cardiac rehabilitation past, present and future: an overview. Cardiovasc. Diagn. Ther. 2, 38-49. https://doi.org/10.3978/j.issn.22233652.2012.01.02

O'Kane, A.A., Mentis, H., 2012. Sharing medical data vs. health knowledge in chronic illness care, in: Proceedings of the 2012 ACM Annual Conference Extended Abstracts on Human Factors in Computing Systems Extended Abstracts - CHI EA '12. ACM Press, New York, New York, USA, p. 2417. https://doi.org/10.1145/2212776.2223812

Patrick D. Savage, Bonnie K. Sanderson, Todd M. Brown, K., Berra, P.A.A., 2012. Clinical Research in Cardiac Rehabilitation and Secondary Prevention: Looking Back and Moving Forward 31, 333-341. https://doi.org/10.1097/HCR.0b013e31822f0f7 9.Clinical

Prince, S.A., Adamo, K.B., Hamel, M.E., Hardt, J., Gorber, S.C., Tremblay, M., 2008. A comparison of direct versus self-report measures for assessing physical activity in adults : a systematic review 24 . https://doi.org/10.1186/1479-5868-5-56

Rauch, B., Davos, C.H., Doherty, P., Saure, D., Metzendorf, M.-I., Salzwedel, A., Vö Ller, H., Jensen, K., Schmid, J.-P., 2016. The prognostic effect of cardiac rehabilitation in the era of acute revascularisation and statin therapy: A systematic review and metaanalysis of randomized and non-randomized studies - The Cardiac Rehabilitation Outcome Study (CROS). Eur. J. Prev. Cardiol. 23, 1914-1939. https://doi.org/10.1177/2047487316671181

Sankaran, S., Frederix, I., Haesen, M., Dendale, P., Luyten, K., Coninx, K., 2016. A Grounded Approach for Applying Behavior Change Techniques in Mobile Cardiac TeleRehabilitation, in: Proceedings of the 9th ACM International Conference on PErvasive Technologies Related to Assistive Environments - PETRA '16. ACM Press, New York, New York, USA, pp. 1-8. https://doi.org/10.1145/2910674.2910680

Schutz, Y., Weinsier, R.L., Hunter, G.R., Weinsier, R.L., 2001. Assessment of Free-Living Physical Activity in Humans : An Overview of Currently Available and Proposed New Measures 9.

Stawarz, K., Cox, A.L., Blandford, A., 2015. Beyond Self-Tracking and Reminders : Designing Smartphone Apps That Support Habit Formation. CHI '15 Proc. 33rd Annu. ACM Conf. Hum. Factors Comput. Syst. 26532662. https://doi.org/10.1145/2702123.2702230

Swain, D.P., Medicine., A.C. of S., Medicine., A.C. of S., 2014. ACSM's resource manual for Guidelines for exercise testing and prescription. Wolters Kluwer Health/Lippincott Williams \& Wilkins, Philadelphia.

Tavella, R., O'Toole, K., Tirimacco, R., Lloyd, A., Pennington, K., Drilling, S., Poulsen, V., Berry, N., Clark, R., Astley, C., Keech, W., 2015. Cardiac rehabilitation referral and completion: results from the South Australian minimum dataset for cardiac rehabilitation programs. Hear. Lung Circ. 24, S451-S452. https://doi.org/10.1016/j.hlc.2015.06.786

Uses, I., Instruments, I., 2005. Guidelines for Data Processing and Analysis of the International Physical Activity Questionnaire ( IPAQ ) Short and Long Forms 1-15.

Vanhees, L., Geladas, N., Hansen, D., Kouidi, E., Niebauer, J., Reiner, Ž., Cornelissen, V., Adamopoulos, S., Prescott, E., Börjesson, M., 
2012. Importance of characteristics and modalities of physical activity and exercise in the management of cardiovascular health in individuals with cardiovascular risk factors: recommendations from the EACPR (Part II). Eur. J. Prev. Cardiol. 19, 1005-1033. https://doi.org/10.1177/1741826711430926

Walters, D.L., Sarela, A., Fairfull, A., Neighbour, K., Cowen, C., Stephens, B., Sellwood, T.,

Sellwood, B., Steer, M., Aust, M., Francis, R., Lee, C., Hoffman, S., Brealey, G.,

Karunanithi, M., 2010. A mobile phone-based care model for outpatient cardiac rehabilitation : the care assessment platform ( CAP ). 